Boletín de la Sociedad Geológica Mexicana

Volumen 61, NúM. 3, 2009, P. 491-497

NOTA TÉCNICA

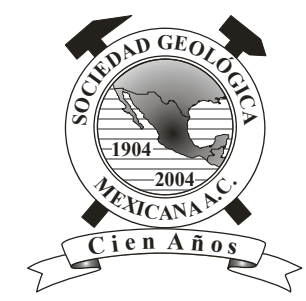

\title{
Una nueva interpretación de la estratigrafía de la Región de Tolimán, Estado de Querétaro
}

\author{
Víctor M. Dávila Alcocer, ${ }^{*}$, Elena Centeno García ${ }^{1}$, Victor Valencia ${ }^{2}$ y Elisa Fitz D. ${ }^{3}$ \\ ${ }^{1}$ Departamento de Geología, \\ Instituto de Geología, Universidad Nacional Autónoma de México, \\ Ciudad Universitaria, México, D. F,. 04510. \\ ${ }^{2}$ Department of Geosciences, University of Arizona. \\ ${ }^{3}$ Department of Geology and Geophysics, University of Minnesota. \\ *davilal@unam.mx
}

\section{Resumen}

En la presente nota se propone que la sucesión de terrígenos más antigua que aflora en la región de Tolimán, sea dividida en dos unidades: El complejo El Chilar, constituido por turbiditas siliciclásticas intensamente deformadas y la Formación San Juan de la Rosa como compuesta por una sucesión volcano-sedimentaria depositada en condiciones marinas. El volcanismo de la Formación San Juan de la Rosa es de composición félsica y de edad Jurásico Tardío-Cretácico Inferior. Se postula como hipótesis de trabajo que el magmatismo félsico de la Formación San Juan de la Rosa sea parte de la migración en tiempo y espacio hacia el sur del arco volcánico jurásico del centro de México.

Palabras clave: Jurásico, Cretácico, arco volcánico, félsico, México

Abstract

It is proposed in this note that the oldest terrigenous rocks cropping out in the Tolimán region in the state of Querétaro belong to two different units: El Chilar complex and the San Juan de la Rosa Formation. The first consists of highly deformed siliciclastic turbidites and the second is a volcano-sedimentary succession deposited in marine conditions. The volcanism of the San Juan de la Rosa Formation is felsic in composition and Late Jurassic-Early Cretaceous in age. We postulate as a working hypothesis that the felsic magmatism of the San Juan de la Rosa Formation are part of the Jurassic volcanic arc in central Mexico that migrated in time and space towards the south.

Key words: Jurassic, Cretaceous, volcanic arc, felsic, Mexico.

\section{Introducción}

La región de Tolimán está localizada al noreste de la Ciudad de Querétaro, en los límites con la Sierra Madre Oriental y el Cinturón Volcánico Transmexicano (Fig.1). Esta región resulta clave para la reconstrucción de la evolución tectónica de México por su estratigrafía y por su posición geográfica, ya que es una de las pocas zonas con afloramientos de rocas pre-cretácicas en el centro de México. La región se encuentra geográficamente en un punto intermedio entre las rocas que registraron una evolución tectónica mesozoica asociada a un proceso de subducción en el margen occidental de Norteamérica y las sucesiones que se originaron por una tectónica extensiva ligada a la evolución del Golfo de México.

Con base en trabajo de campo, se encontraron evidencias importantes para la separación de las rocas de la región en unidades litoestratigráficas distintas a las que se habían propuesto por autores previos (Carrillo-Martínez y Suter, 1982; Chauve et al., 1985; Carrillo-Martínez, 2000). En esta nota se reportan datos nuevos de campo de la porción basal de la columna estratigráfica de la región de Tolimán, que apoyan la división en dos unidades terrígenas en lugar de una, y sus probables implicaciones regionales (Fig. 2). 


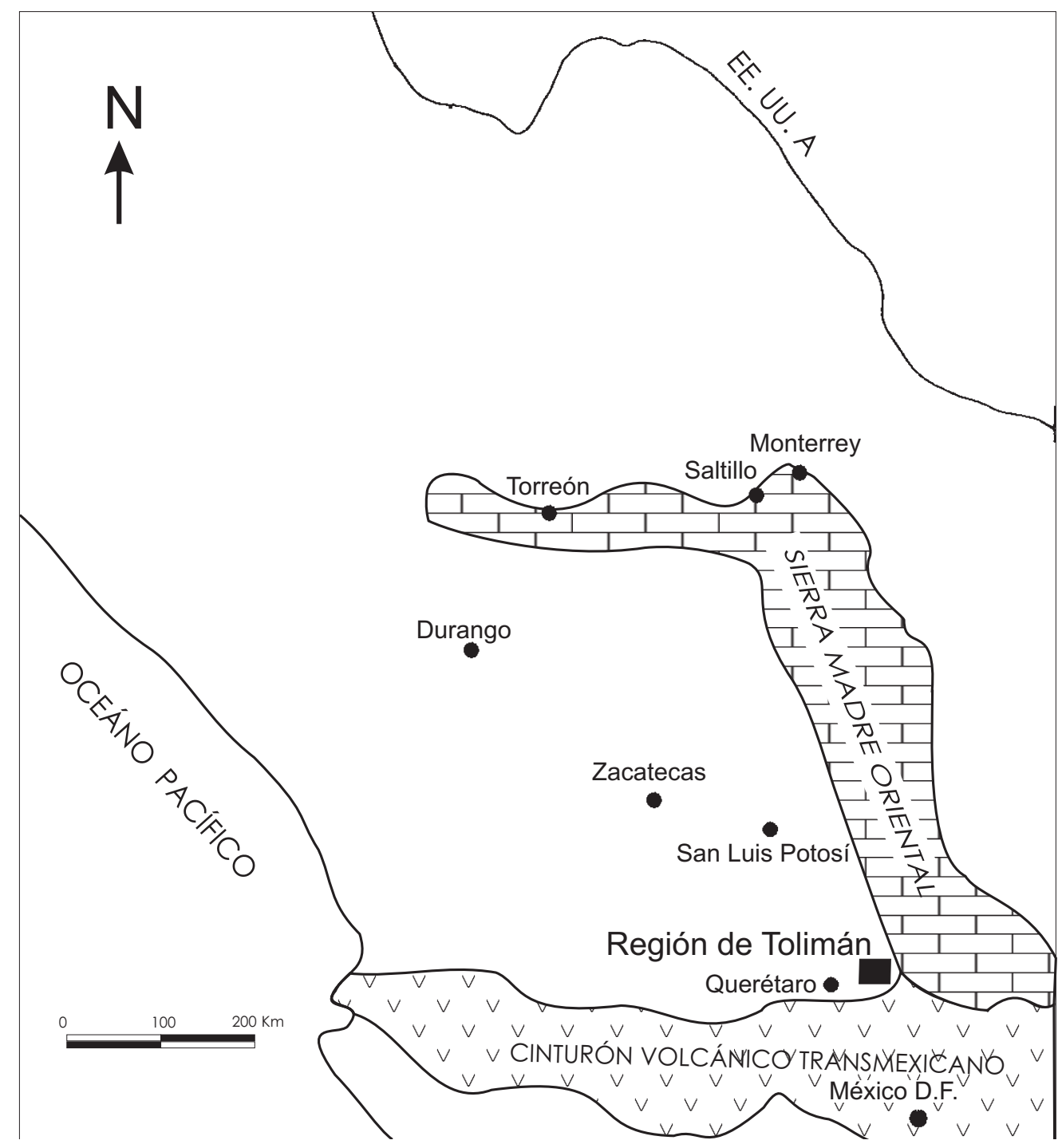

Fig. 1 Mapa de localización de la región de Tolimán, estado de Querétaro.

\section{Estratigrafía}

Existe discrepancia en la sucesión estratigráfica propuesta por diversos autores para el área de estudio, principalmente en lo referente a la definición y la edad correspondiente a las unidades estratigráficas que subyacen a la secuencia calcárea del Cretácico Inferior (Segerstrom, 1961; Carrillo-Martínez y Suter, 1982; López-Ramos, 1985; Chauve et al., 1985; Carrillo-Martínez, 2000).

Segerstrom (1961) y López-Ramos (1985) describieron rocas "antiguas" en la región de Tolimán, las cuales fueron consideradas por López Ramos (1985) tentativamente como de edad Paleozoica, nombrándola informalmente como formación El Chilar. En cambio, Carrillo (2000) agrupó a todas las rocas que subyacen a las formaciones Peña Azul y Tamaulipas del Cretácico Inferior-medio en una sola unidad, con el nombre de Formación San Juan de la Rosa.
Nuestras observaciones de campo sugieren que las rocas en cuestión pertenecen a dos paquetes litológicos muy distintos, separados por una discordancia angular (Figura 3).

Las rocas más antiguas están constituidas por cuarzo arenitas y lutitas ricas en cuarzo. Dichas rocas contienen bloques de tamaño diverso ( 500 metros a pocos centímetros) de la misma arenisca, y un bloque de gran dimensión de pedernal que contiene rocas ígneas básicas. Los bloques y matriz se encuentran intensamente deformados llegando a formar zonas de melage tectónico, localmente metamorfizados a facies muy baja de esquistos verdes. En lo sucesivo, a esta unidad, le denominaremos complejo El Chilar. Se desconoce la edad de depósito y de deformación de este complejo, las edades $\mathrm{U} / \mathrm{Pb}$ obtenidas de circones detríticos contenidos en una muestra de arenisca sugieren que la edad de depósito es al menos post-pérmica y pre-Jurásico Tardío (Dávila-Alcocer et al., 2008). 


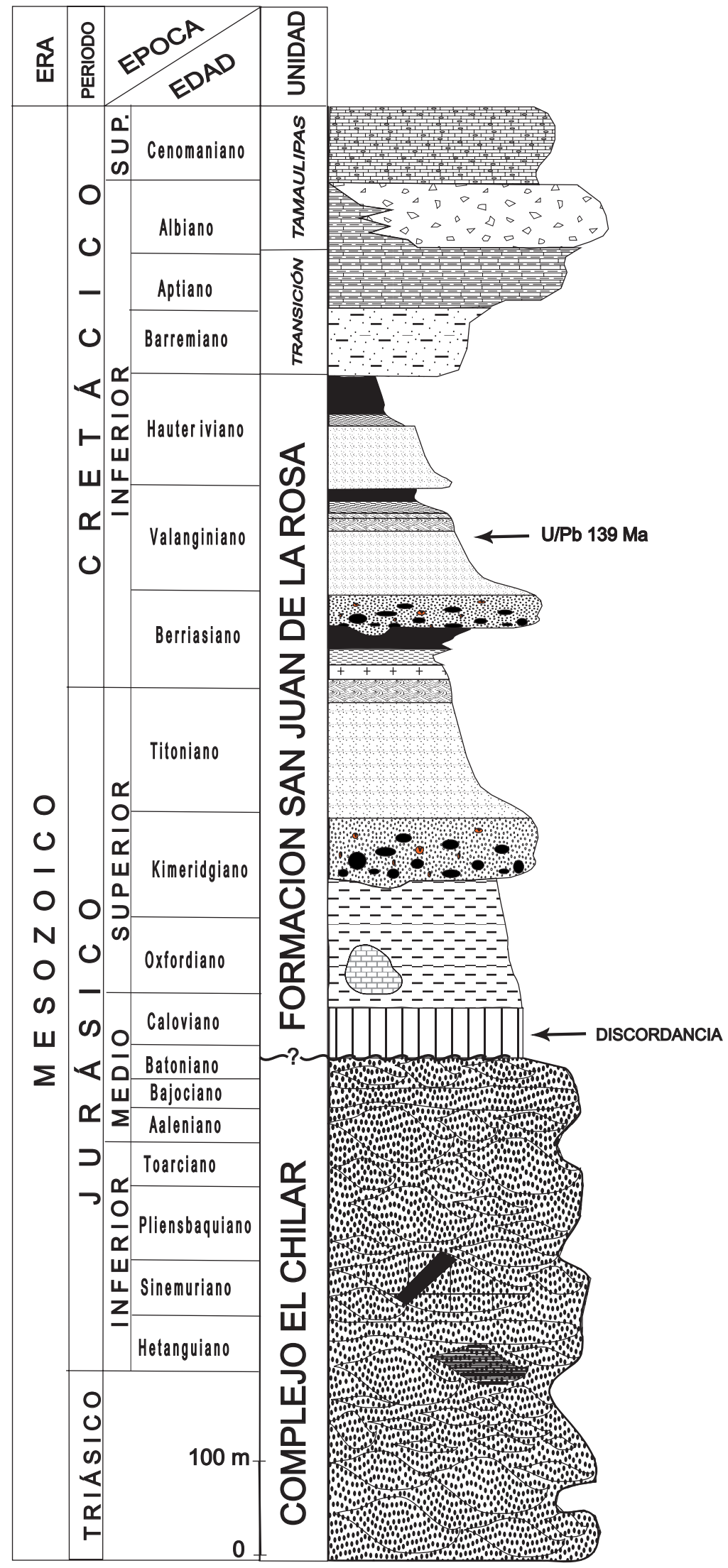

Fig.2 Columna estratigráfica generalizada de la región de Tolimán 

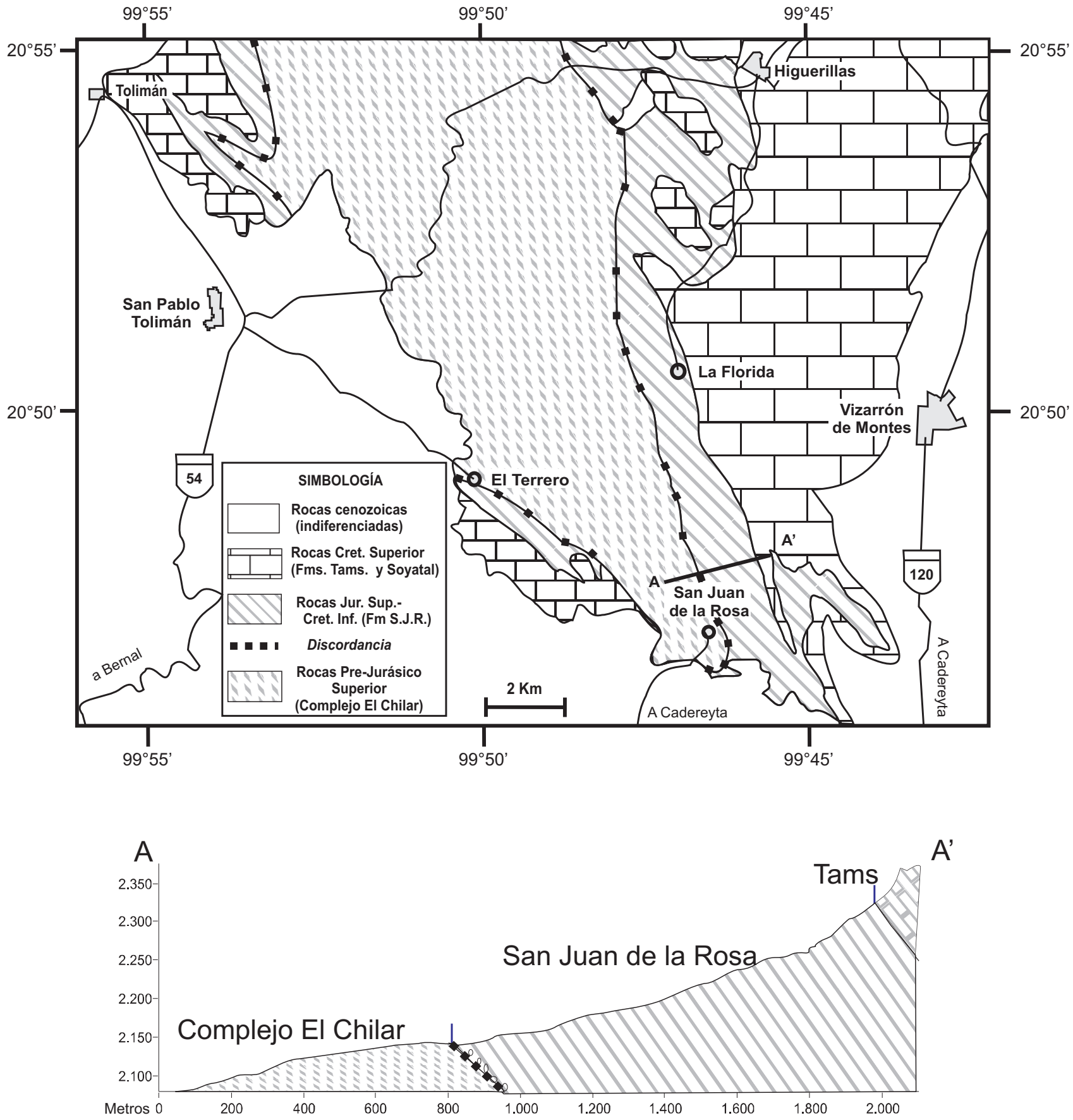

Fig. 3 Mapa donde se muestra el área de afloramiento del complejo El Chilar y de las rocas jurásico-cretácicas de la Formación San Juan de la Rosa, así como su relación con la secuencia suprayacente. (modificado de Carrillo-Martínez, 2000). Además, sección estratigráfica modelo de la Región de Tolimán

Sobre el complejo El Chilar descansa discordantemente una sucesión volcano-sedimentaria a la que restringimos el nombre de Formación San Juan de la Rosa (Fig. 2, 3). En esta última, Martínez-Hernández (1979) reporta amonites de edad Kimeridgiano Tardío - Titoniano Temprano. Sin embargo, dentro de esta unidad, obtuvimos una edad U/Pb del Cretácico Temprano (139 Ma) en zircones recuperados de una muestra de litoarenita volcánica (Dávila-Alcocer et al., 2008).
Esto indica que al menos parte del depósito se realizó durante este tiempo. Esta formación cambia de manera transicional a una sucesión de limolitas calcáreas con lutitas y calizas de la Formación Peña Azul, del Cretácico, la cual ocasionalmente incluye flujos de escombros y calizas con pedernal.

La Formación San Juan de la Rosa ya había sido reportada como una sucesión volcaniclástica por Chauve et al. 


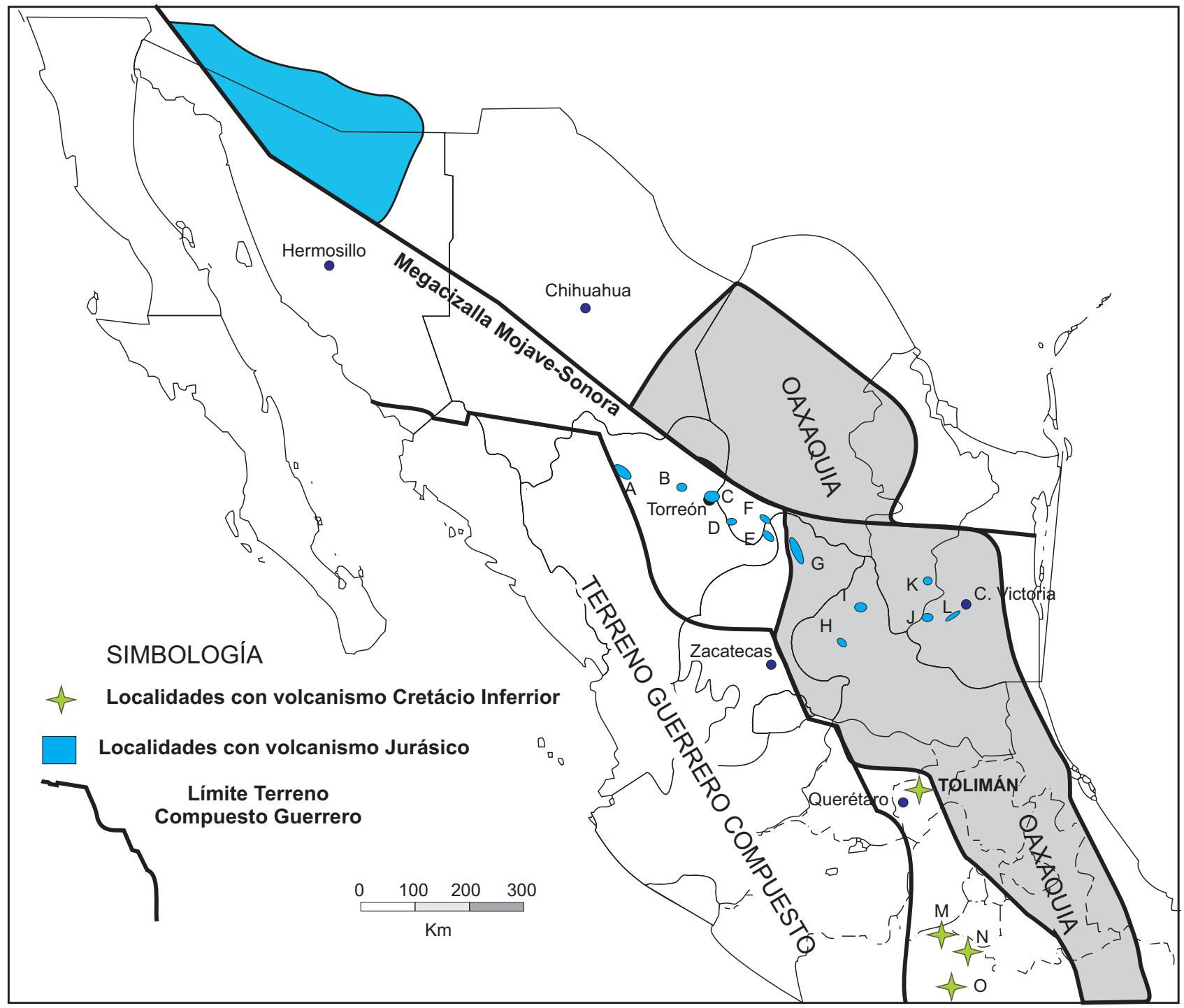

Fig. 4 Afloramientos de rocas volcánicas del Jurásico al norte de la Megacizalla Mojave-Sonora y las correspondientes desplazadas hacia el sureste. (A.- Santa María del Oro, B.- San Pedro el Gallo C.- Villa Juárez, D.- Sierra de Jimulco, E.- Sierra de Ramírez, F.- Cañón de Ahuichila, G.- Caopas-Pico de Teyra, H.- Charcas, I.- Sierra de Catorce, J.- Miquihuana, K.- Aramberri, L.- Cañón de Huizachal). (modificado de Jones, et al., 1995) , y áreas con volcanismo Jurásico Tardío-Cretácico Temprano, Tolimán, M.-Taxco, N.- Zicapa, O.- Chapolapa.

(1985). En campo se observó que gran parte de tal formación está constituida por productos primarios de un volcanismo félsico submarino. La columna estratigráfica consiste principalmente de areniscas y conglomerados, ambos constituidos por clastos volcánicos, alternados con lutitas. Estas rocas presentan estructuras primarias típicas de depósitos turbidíticos, tales como gradación, intraclastos, estratificación cruzada, estratificación convoluta, estructura en flama, y pliegues sinsedimentarios, entre otras. La presencia de amonitas y las estructuras primarias sugieren depósitos de tipo epiclástico. La unidad incluye flujos piroclásticos y derrames riolíticos, cuya petrología a detalle, así como su caracterización geoquímica aún están en proceso, sin embargo consideramos interesante adelantar el reporte de estas rocas en la presente nota.
La Formación San Juan de la Rosa fue interpretada por Chauve et al. (1985) como parte del "dominio" cordillerano, quienes la asociaron con el Arco Alisitos de la Península de Baja California. Sin embargo esta correlación directa no es factible, ya que entre la Formación San Juan de la Rosa y el Arco Alisitos se encuentra el Terreno Compuesto Guerrero (Centeno-García, 2005, Centeno-García et al., 2008), el cual tiene una estratigrafía compleja y muy distinta de la observada en la región de Tolimán.

Por otra parte, la Formación San Juan de la Rosa descansa discordantemente sobre sucesiones de cuarzo arenitas y lutitas ricas en cuarzo del complejo El Chilar que son muy similares a las unidades siliciclasticas que forman el basamento de las secuencias volcánicas del centro de México tales como la Formación La Ballena, (Centeno-García et al., 
1997; Silva-Romo et al., 2000) y Formación Taray (DíazSalgado, et al., 2003, Díaz-Salgado, 2004, Anderson et al., 2005).

La ubicación de las áreas de afloramiento de las rocas con una composición y estratigrafía similar a la de la Formación San Juan de la Rosa en la región de Tolimán se presenta en la Figura 4. Las formaciones volcánicas-volcaniclásticas del centro de México a las que se hace referencia en el párrafo anterior son Nazas, Caopas, Rodeo, y Huizachal (Barboza et al., 2007; Jones et al., 1995), marcadas con las letras A a la L en el mapa de la figura 4. Todas ellas de composición riolítica-andesítica y cuyas edades radiométricas son: Caopas (158 Ma, Jones et al., 1995), Rodeo (183 Ma, LópezInfanzón, 1986), Huizachal (193 Ma, Barboza-Gudiño et al., 2007, 174.7 Ma., Barboza-Gudiño et al., 2004). Sin embargo, el magmatismo de Tolimán alcanza rangos más jóvenes de edad (139 Ma, Dávila et al., 2008) hacia la cima, y amonitas del Kimeridgiano-Titoniano hacia la base.

Por otra parte hay abundante magmatismo del Cretácico Inferior en el sur del país (Centeno-García et al., 2008), con una composición similar a la del volcanismo de la Formación San Juan de la Rosa, estas son Roca Verde Taxco Viejo y Esquistos Taxco, formaciones Zicapa y Chapolapa (Fig. 4). Las unidades en Taxco, están constituidas por lavas riolíticas y andesíticas acompañadas por tobas de la misma composición interestratificadas con areniscas y lutitas ricas en cuarzo depositadas en condiciones submarinas. Mientras que en Chapolapa está compuesta principalmente por flujos de lavas riolíticos, dacíticos y escasos andesíticos así como rocas epiclásticas (García-Díaz, 2004). En cambio la Formación Zicapa consiste de depósitos continentales intercalados con productos volcánicos de composición andesítica. Aunque se desconoce el basamento de dichas rocas (Talavera-Mendoza 1993).

Todas estas unidades volcánicas comparten las siguientes características a) el carácter félsico del magmatismo, y b) que todas estas formaciones volcánicas-volcaniclásticas están cubiertas por gruesas secuencias calcáreas del Cretácico medio Superior y que son parte de los mares epicontinentales de Norteamérica.

En esta nota se propone como hipótesis de trabajo que las rocas félsicas jurásico-cretácicas de la Formación San Juan de la Rosa probablemente formaron parte de un cinturón de volcanismo félsico que se extiende a lo largo de la región occidental de Oaxaquia, cuyas edades aparentemente migran de Jurásico Temprano-Medio (193-158 Ma; Jones et al., (1995) en el centro de México al Jurásico Tardío-Cretácico Temprano en Tolimán (139 Ma, Dávila et al., 2008) y hasta el Cretácico Temprano (Taxco 130-131 Ma, Chapolapa 129$133 \mathrm{Ma}$; Campa e Iriondo, 2004; y Zicapa 127 Ma, Fitz et al., 2002) en el sur de México. Con la diferencia de que el volcanismo en la región central de México es continental, mientras que en Tolimán, Taxco y Chapolapa se desarrolló en condiciones submarinas.

\section{Agradecimientos}

Este trabajo contó con el apoyo financiero de los proyectos de investigación Nos. IN109605, IN115208 PAPIIT (DGAPA, UNAM).

\section{Referencias}

Anderson, T.H., Jones, N. W. McKee, J.W., 2005, The Taray Formation: Jurassic (?) mélange in northern Mexico-Tectonic implications, Geological Society of America Special Paper 393, p 427-455.

Barboza-Gudiño, J.R., Orozco-Esquivel, A.T., Gómez-Anguiano, M., Zavala-Monsiváis, A., 2007, The Early Mesozoic volcanic arc of western North America in northeastern Mexico: Journal of South American Earth Sciences, 25 (1), 49-63.

Barboza-Gudiño, J.R., Hoppe, M., Gómez-Anguiano, M., MartínezMacías, P.R., 2004, Aportaciones para la interpretación estratigráfica y estructural de la porción noroccidental de la Sierra de Catorce San Luis Potosí, México: Revista Mexicana de Ciencias Geológicas, 21(3), 299-319.

Campa, M.F., and Iriondo, A., 2004, Significado de dataciones Cretácicas de los arcos volcánicos de Taxco, Taxco Viejo y Chapolapa, en la evolución de la plataforma Guerrero-Morelos: Unión Geofísica Mexicana: Reunión Nacional de Ciencias de la Tierra, GEOS, v. 24, p. 173 .

Carrillo-Martínez., M., 1998 (2000), Resumen de la geología de la Hoja Zimapán, estados de Hidalgo y Querétaro, escala 1:100,000: México, D.F. Universidad Nacional Autónoma de México, Instituto de Geología, Carta Geológica de México, Serie 1:100,000, 1 mapa con texto.

Carrillo-Martínez, M., Suter, M., 1982, Tectónica de los alrededores de Zimapán, Hidalgo y Querétaro: México, D.F., Sociedad, Geológica Mexicana, Libro-guía de la excursión geológica a la región de $\mathrm{Zi}$ mapán y áreas circundantes, p. 1-20.

Centeno-García, E. 2005, Review of Upper Paleozoic and Lower Mesozoic stratigraphy and depositional environments of central and west Mexico: Constraints on terrane analysis and paleogeography: Geological Society of America Special Paper 393. p. 233-258.

Centeno-García E., Guerrero-Suastegui M., Talavera-Mendoza O., 2008, The Guerrero Composite Terrane of western Mexico: Collision and subsequent rifting in a supra-subduction zone. The Geological Society of America Special Paper 436 p. 279-308.

Centeno-García, E., Silva-Romo, G., 1997, Petrogenesis and tectonic evolution of central Mexico during Triassic-Jurassic time: Revista Mexicana de Ciencias Geológicas, 14, 244-260

Chauve, P., Fourcade, E., Carrillo-Martínez, M., 1985, Les rapports structuraux entre les domines cordillérain et mésogéen dans la partie centrale du Mexique: Comptes Rendus de l'Académie des Sciences, t. 301, série II, no. 5 p. 335-340.

de Cserna, Z., 1965. Reconocimiento geológico de la Sierra Madre del Sur de México, entre Chilpancingo y Acapulco, Estado de Guerrero. UNAM, Instituto de Geología, Boletin, v. 62, pp.1-76.

de Cserna, Z., Fries, C. Jr., 1981, Hoja Taxco 14Q-h(7), y Resumen de la Geología de la Hoja Taxco, Estados de Guerrero, México y Morelos, Instituto de Geología UNAM, Carta Geológica de México, Series, mapa $(1: 100,00)$ y texto, $47 \mathrm{p}$.

Dávila-Alcocer, V.M., Centeno-García, E., Barboza-Gudiño R., Valencia, V., Fitz-Díaz, E., 2008, Detrital Zircon Ages from the El Chilar Accretionary Complex and Volcaniclastic Rocks of the San Juan De La Rosa Formation, Toliman, Queretaro, Mexico: (resumen) in Geological Society of America Abstracts with Programs, Vol. 40, No. 6, p. 198

Díaz-Salgado, C., 2004, Caracterización tectónica y procedencia de la Formación Taray, Región de Pico de Teyra, Estado de Zacatecas: México, D.F., Universidad Nacional Autónoma de México, Instituto de Geología, Posgrado en Ciencias de la Tierra, tesis maestría. 
Díaz-Salgado, C., Centeno-García, E., Gehrels, G., 2003, Stratigraphy, depositional environments, and tectonic significance of the Taray Formation, northern Zacatecas state, Mexico: (resumen) in GSA 99th Cordilleran Section Annual Meeting, Abstracts with Programs, v. 35 , n. 4 , p. 71 .

Fitz, D.E., Campa, M.F., y López, M.M., 2002, Fechamiento de lavas andesíticas de la Formación Zicapa, en el límite oriental de la Plataforma Guerrero Morelos, Actas INAGEQ, v. 8, p. 178.

Jones, N., W.J., McKee, J.W., Anderson, T.H., Silver, L.T., (1995) Jurassic volcanic rocks in northeastern Mexico: A possible remnant of a Cordilleran magmatic arc: Geological Society of America Special Paper 301, p. 179-190.

López-Infanzón, M., 1986, Estudio petrogenético de las rocas ígneas en las formaciones Huizachal y Nazas: Boletín de la Sociedad Geológica Mexicana, 47(2), 1-42.
López-Ramos E., 1985, Geología de México (3ª ed.), tomo II: México D.F. edición particular.

Martínez-Hernández, S., 1979, Contribución al estudio geológico del sector Vizarrón-Tolimán, estado de Querétaro, México: México D.F., Instituto Politécnico Nacional, Escuela Superior de Ingeniería y Arquitectura, tesis profesional.

Segerstrom, K., 1961, Geology of the Bernal-Jalpan area, Edo. de Queretaro, Mexico: U.S. Geol. Survey, Bulletin 1104-B, p. 19-85.

Silva-Romo, G., Arellano-Gil, J., Mendoza-Rosales, C., Nieto-Obregón, J., 2000, A submarine fan in the Mesa Central, Mexico: Journal of South American Earth Sciences 13, 429-442.

Talavera-Mendoza O., 1993, Les formations orogeniques mesozoiques du Guerrero (Mexique meridional). Contribution a la connaissance de l'evolition geodinamique des cordilleres mexicaines. Universite Joseph Fourier-Grenoble I. Tesis doctoral

Manuscrito recibido: 29/09/2008

Manuscrito corregido: 10/03/2009

Manuscrito aceptado: 20/07/2009 